\title{
1 Complex genetic architecture underlying the plasticity of maize agronomic traits
} 2

3 Minliang $\mathrm{Jin}^{1}$, Haijun $\mathrm{Liu}^{2}$, Xiangguo $\mathrm{Liu}^{3}$, Tingting Guo ${ }^{1,4}, \mathrm{Jia} \mathrm{Guo}^{3}$, Yuejia Yin ${ }^{3}$, Yan $\mathrm{Ji}^{5}$, 4 Zhenxian $\mathrm{Li}^{6}$, Jinhong Zhang ${ }^{6}$, Xiaqing Wang ${ }^{1}$, Feng Qiao ${ }^{1}$, Yingjie Xiao ${ }^{1,4}$, Yanjun Zan ${ }^{7 *}$,

5 Jianbing $\operatorname{Yan}^{1,4^{*}}$

$7{ }^{1}$ National Key Laboratory of Crop Genetic Improvement, Huazhong Agricultural University,

8 Wuhan, 430070, China

$9{ }^{2}$ Gregor Mendel Institute, Austrian Academy of Sciences, Vienna BioCenter, 1030, Vienna,

10 Austria

$11{ }^{3}$ Institute of Agricultural Biotechnology, Jilin Academy of Agricultural Sciences, Changchun,

12 130033, China

$13{ }^{4}$ Hubei Hongshan Laboratory, Wuhan, 430070, China

$14{ }^{5}$ College of Life Sciences, Sichuan University, Chengdu, 610065, China

$15{ }^{6}$ Institute of Agricultural Sciences of Xishuangbanna Prefecture of Yunnan Province,

16 Jinghong, 666100, China

$17{ }^{7}$ Umeå Plant Science Center, Department of Forestry Genetics and Plant Physiology,

18 Swedish University of Agricultural Sciences, Umeå, 90736, Sweden

$20 *$ Corresponding authors

21 Jianbing Yan,

22 Email: yjianbing@mail.hzau.edu.cn

23 Yanjun Zan,

24 Email: yanjun.zan@slu.se 


\section{Abstract}

26 Phenotypic plasticity is the property of a given genotype to produce multiple phenotypes in 27 response to changing environmental conditions. Understanding the genetic basis of 28 phenotypic plasticity and establishing a predictive model is highly relevant for future 29 agriculture under changing climate. Here, we report findings on the genetic basis of 30 phenotypic plasticity for 23 complex traits using a maize diverse population, planted at five 31 sites with distinct environmental conditions and genotyped with $\sim 6.60$ million SNPs. We 32 found that altitude-related environmental factors were main drivers for across site variation in 33 flowering time traits but not plant architecture and yield traits. For 23 traits, we detected 109 34 QTLs, of which 29 was for mean, 66 was for plasticity, and 14 for both parameters, besides, $3580 \%$ of the QTLs were interreacted with the environment. The effects of several QTLs 36 changed in magnitude or sign, driving variation in phenotype plasticity, and we further 37 experimentally validated one plastic gene ZmTPS14.1 whose effect was likely mediated by 38 the compensation effect of ZmSPL6 which was from the downstream pathway probably. By 39 integrating genetic diversity, environmental variation, and their interaction in a joint model, 40 we could provide site-specific predictions with increased accuracy by as much as $15.5 \%$, $413.8 \%$, and $4.4 \%$ for DTT, PH, and EW, respectively. Overall, we revealed a complex genetic 42 architecture involving multiallelic, pleiotropy, and genotype by environment interaction 43 underlying maize complex trait mean and plasticity variation. Our study thus provided novel 44 insights into the dynamic genetic architectures of agronomic traits in response to changing 45 environments, paving a practical route to precision agriculture.

47 Keywords: Complex traits, Phenotype plasticity, QTL by environment interaction, 48 Crop improvement, Zea mays 


\section{Introduction}

50 Upon climate change, plants display plastic response, where a single genotype produces 51 multiple phenotypes through changes in gene expression, physiological and morphological 52 levels $1^{1,2}$. Such plastic response (phenotype plasticity) was also described as genotype by 53 environment interaction (G-by-E) ${ }^{3-5}$, with organisms changing their performance across 54 environments, releasing heritable variation ${ }^{6-9}$ that are highly relevant in complex trait 55 variation and adaptation ${ }^{4,10-12}$. In the context of crop breeding, one strategy is to minimize 56 plasticity or G-by-E interaction by using the best linear unbiased prediction value (BLUP), 57 making developed cultivar broadly applicable to a wide range of environments ${ }^{13}$. 58 Alternatively, performance could be maximized in individual environments by enriching site59 specific beneficial alleles that are either neutral or unfavourable at other sites ${ }^{12,14}$. This is 60 similar to what natural selection have acted on wild populations, where local adaptation has 61 resulted in genotypes with optimized phenotypes at their native environments that are often 62 maladapted in new environments ${ }^{15-18}$.

63 Increased plasticity may represent the future of crop breeding and biodiversity management 64 in the light of climate change, as such strategy confers high resilience genotypes for future 65 challenges while achieving optimal phenotype locally. To achieve this goal, efforts have been 66 made to study the genetic architecture of plasticity ${ }^{19-22}$ and dissect the underlying QTLs ${ }^{4,11,23-}$

$67{ }^{26}$. Studies in maize have revealed both similarity and difference in the genetic architectures 68 of trait mean and plasticity ${ }^{24,25}$, suggesting breeders could manipulate trait mean and 69 plasticity semi-independently to meet the challenge of feeding the growing population.

70 Further investigations demonstrated the role of plastic QTLs in heterosis and adaptation from

71 tropical to temperate zone, paving the way to genomic-promised crop improvement by 72 manipulating the phenotypic plasticity ${ }^{27,28}$.

73 Despite the insights gained through these efforts, several questions remain elusive. First, 74 there is a lack of understanding of the dynamics of complex traits genetic architectures across 75 environments, such as the impact of specific environmental factors on range-wide complex 76 trait variation, how dynamic are the genetic architectures of agronomic traits over major 77 production zone? What alleles are favoured at each production site? Whether they have 78 genetic effects on multiple traits with antagonistic pleiotropy? How much genetic gain could 79 be achieved by exploiting these alleles? 
80 Second, in Fisher's decomposition of phenotype mean, the environmental effect is a

81 combinatory effect from multiple environmental factors, such as temperature, day length, and 82 soil conditions, etc. With an increased ability to quantify air and soil conditions using 83 developments in remote sensing, it is of great interest to decompose the combinatory 84 environment effects into effects from concrete environmental factors and study their impact 85 on complex trait variation and prediction. Last but not least, plasticity was often treated as a 86 composite index ${ }^{19-22}$, neglecting the fact that plasticity is environment-dependent, being 87 variable when quantified using different combinations of environments. With a growing 88 number of environments that we could investigate, it is worthwhile to differentiate plasticity 89 quantified using an overall index and refine plasticity measures from specific combinations 90 of environments.

91 To provide a deeper insight into these questions, we developed the Complete-diallel plus 92 Unbalanced Breeding-derived Inter-Cross (CUBIC) population of 1404 advanced inter-cross 93 lines from 24 representative breeding founders ${ }^{29}$ and studied the variation of 23 key 94 agronomic traits at five sites spanning China's major summer maize production zone (Fig. 95 1A) from northeast at Jilin (JL; N $43^{\circ} 42^{\prime}$, E $\left.125^{\circ} 18^{\prime}\right)$ to central plains at Henan (HN; N $35^{\circ}$ $9627^{\prime}$, E $114^{\circ} 01^{\prime}$ ). We revealed major contributions from the latitude-related environmental 97 factors to across site phenotypic variation for flowering time traits but not for others. And we 98 dissected the within and across environment variation to 109 QTLs with complex genetic 99 architectures involving multiallelic, pleiotropy, and genotype by environment interaction. In 100 particular, we found that extensive QTL by environment interaction and dynamic in mean 101 QTL effects across environments was driving the variation in phenotype plasticity. A joint 102 model with site-specific predictions and higher accuracy was developed by integrating 103 genetic diversity, environmental variation, and their interaction, paving a way to genomics104 directed maize improvement.

107 The impact of clinal variation in environmental factors on the mean and plasticity of 23 108 maize complex traits

109 We surveyed the performance of 23 traits across five sites spanning Chinese major summer 110 maize production zone with longitudinal variation from E114 $01^{\prime}$ (Henan; $\mathrm{HN}$ ) to E125 $18^{\prime}$

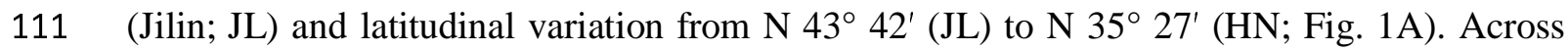


112 the five sites, daily highest temperature (TemH), daily temperature difference (TemD), and 113 day length (DayL) varied significantly (Fig. 1B, C). Nearly all the traits (22 out of 23, except 114 for Leaf number below ear, LNBE) were significantly correlated with latitude at the five sites, 115 suggesting a general contribution from spatially variable environmental factors to maize 116 agronomic trait variation (Fig. 1D-F; Figure S1; Table S1, S2). Flowering time traits (days to 117 tassel DTT; days to silking, DTS; days to anthesis, DTA) displayed the strongest latitudinal 118 variation with trait median measured at JL being 1.5 times larger than that $\mathrm{HN}$ (Figure S1; 119 Table S2). Unlike flowering time, clinal variation in plant architecture traits (Plant height, $\mathrm{PH}$; 120 Ear height, EH; Ear leaf width, ELW) and yield traits were weaker, being more distinctive 121 between the northern (JL, LN, and BJ) and southern ( $\mathrm{HB}$ and $\mathrm{HN}$ ) sites (Fig. 1B, C; Figure $122 \mathrm{~S} 1)$.

123 To explore how the 23 traits responded to the across-site environmental perturbation, we first 124 rank-transformed each trait measured at individual sites and quantified the phenotype 125 plasticity as coefficient variation of rank (VarR) across the five sites. All the 23 traits 126 displayed variation in phenotype plasticity (Fig. 1G), and yield traits were more plastic than 127 flowering time and plant architecture traits. Contributions from environment (E) and 128 genotype by environment interaction (G-by-E) varied significantly among the three 129 categories of traits. For example, TemH was the major driver for (median $=84.2 \%$ ) across130 site variation of flowering time traits (DTT, DTA, and DTS; Fig. 1I), while its contribution to 131 the variation of remaining traits was much lower (Fig. 1I; media=9\%). In contrast, G-by-E 132 made a higher contribution (median $=32.8 \%$; Fig. 1I) to the across site variation of yield 133 traits, being consistent with the observation that the proportions of non-additive variance for 134 yield traits were also higher than that for flowering and architecture traits (Fig. 1H). 135 Altogether, these results illustrated a general contribution from environment factors (TmpD, $136 \mathrm{TmpH}$, and DayL) and their interaction with genotype to the variation of maize complex 137 traits, where the contribution from G-by-E was more prominent for yield traits, indicating the 138 importance and potential value of studying plasticity for yield improvement. 

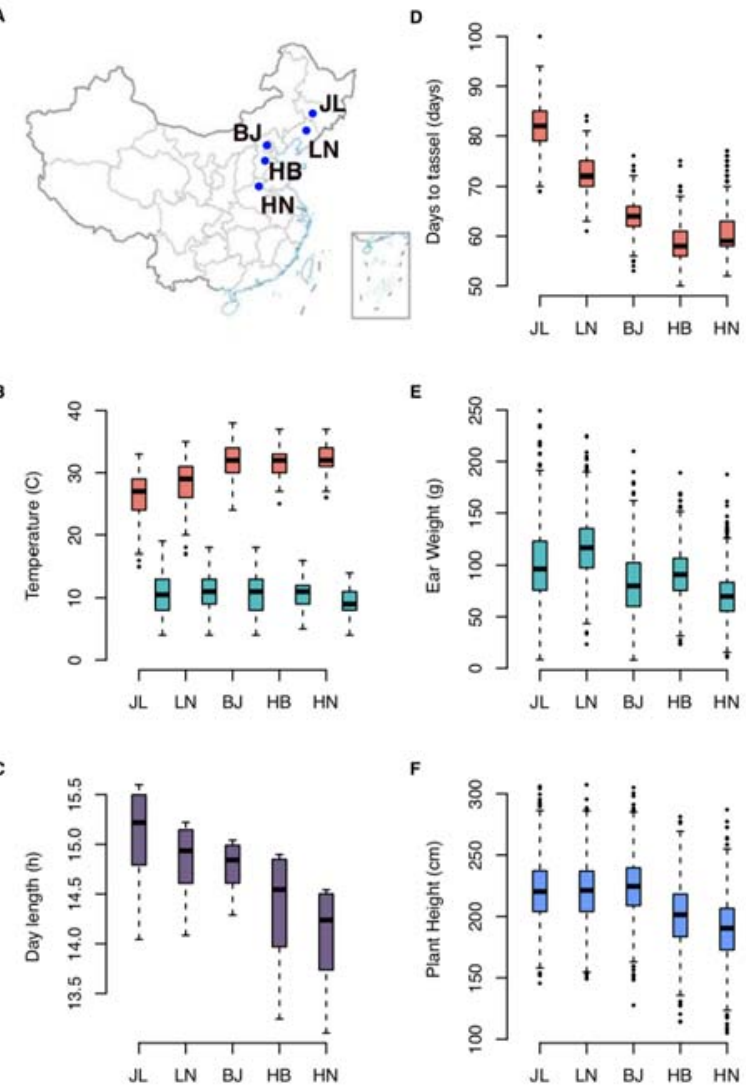

$E$

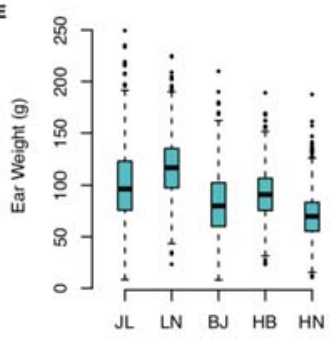

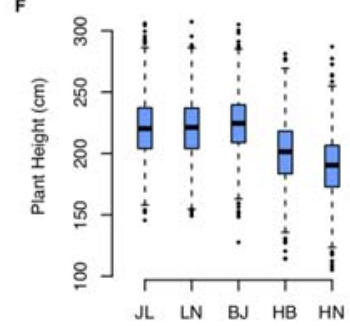

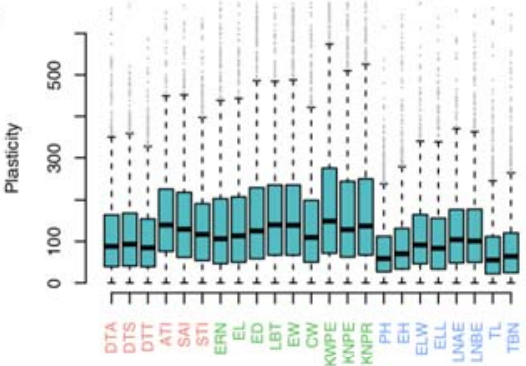

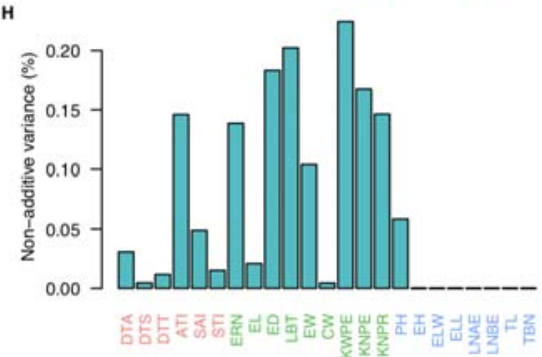

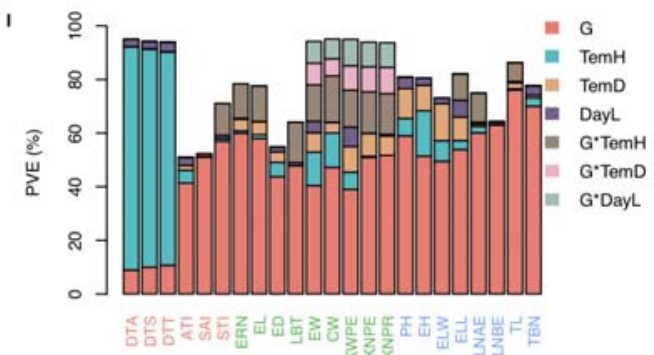

Fig. 1 Environmental variation across China's major summer maize production zone and their impact on the across-site variation of maize complex traits. A) The five surveyed sites spanning China's major maize production zone, where 23 agronomic traits were phenotyped for 1404 inbred lines. B) Boxplot illustrating the highest daily temperature (TemH; coloured in cyan) and daily temperature difference (TemD; coloured in tomato) from sowing to flowering at the five sites. C) Boxplot of the day length (DayL) from sowing to flowering at the five sites. D) Boxplot of Days to tassel (DTT; coloured in tomato), E) Ear weight (EW; coloured in green) and F) Plant height (PH; coloured in cyan) measured at the five sites. G) Boxplot of the phenotype plasticity measured as a coefficient variation of the rank across s (Materials and Methods). The 23 traits (labelled in X-axis) were grouped into 3 categories, flowering traits highlighted in tomato, plant architecture traits labelled in green and yield traits labelled in blue. $\mathbf{H})$ Bar plot of the proportion of non-additive variance (differences between broad-sense heritability, capturing the additive and non-additive effect, and narrow-sense heritability, capturing only the additive effect). Each vertical bar represents a trait, and the height of the bar is proportional to the difference between corresponding broad and narrow-sense heritability. I) Contribution from genotype, the three environmental factors (TemH, TemD and DayL), and their interactions to the across-site variation of the 23 agronomic traits. Each vertical bar represents a trait with the corresponding trait name labelled in $\mathrm{x}$-axis. The coloured segments within each bar represent the contribution from $\mathrm{G}$, TemD, TemH, DayL, and their interaction with $G$ as indicated in the legend. The height of the segment is proportional to the variance explained (PVE) by the corresponding variance component. 
163 Dynamic and complex genetic architecture underlying maize agronomic traits mean and 164 plasticity

165 For each of the 23 traits, we derived two types of measures to quantify the phenotype 166 plasticity, where type I included 10 measures $^{30,31}$ calculated as pairwise difference among 167 five sites to capture specific plasticity (SP), and type II included 4 measures representing 168 overall plasticity (OP): coefficient of variation from raw $(\mathrm{CV})^{30}$, rank transformed data 169 (VarR) ${ }^{30}$, second principal component (PC2) ${ }^{30}$, and Finlay-Wilkinson regression (FWR) ${ }^{32}$ 170 (Figure S2; Materials and Methods). Together with trait mean value from five sites (Mean) 171 and BLUP, these four types of measures (SP, OP, Mean, and BLUP) were used to scan for 172 QTLs underlying trait mean and plasticity, using genome-wide association analysis with 6.6 $173 \mathrm{M}$ genetic polymorphisms (Materials and Methods). In the following section, we first 174 illustrated the results from DTT as an example and then expanded to results from all 23 traits. 175 Hereafter, the 4 types of measures were referred to as $\mathrm{DTT}_{\mathrm{BLUP}}, \mathrm{DTT}_{\mathrm{x}}$ (mean measured at site $176 \mathrm{X})$, SP-DTT $\mathrm{x-y}$ (Specific plasticity measured as $\mathrm{DTT}_{\mathrm{x}}-\mathrm{DTT}_{\mathrm{y}}, \mathrm{X}$ and $\mathrm{Y}$ was site name), and $177 \mathrm{OP}_{\mathrm{DTT}}$ (Overall plasticity calculated using method $\mathrm{z}, \mathrm{z}$ was described in Materials and 178 Methods).

179 Loci associated with the variation of mean and plasticity measures for days to tassel 180 Dynamic QTL effects across environments lead to variation in plasticity

181 A total of 15 QTLs were identified, including 11 QTLs for SP/OP-DTT and 7 QTLs for $182 \mathrm{DTT}_{\text {mean }} / \mathrm{DTT}_{\mathrm{BLUP}}$ with 3 overlaps (Fig. 2A-D, QTLs were obtained by grouping independent 183 SNPs within defined physical distance, Materials and Methods; Table S3, S4, S5). A majority

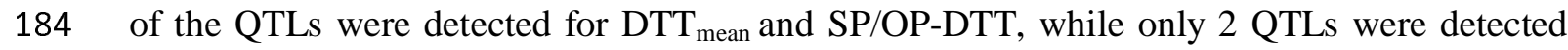
185 for $\mathrm{DTT}_{\mathrm{BLUP}}$, highlighting the added value to analyse $\mathrm{DTT}_{\text {mean }}$ and the derived plasticity 186 measurements individually (Fig. 2B). By contrasting genetic effects of QTLs across sites, two 187 types of QTLs, whose effects changed in magnitude or direction, were detected with 188 significant contribution to the variation of DTT plasticity. For example, different genotypes 189 of QTL8 (chromosome 5: 6,462,711 bp, Fig. 2A-C, E, $667.2 \mathrm{~kb}$ upstream of ZmPHYC2, 190 GRMZM2G129889, a homology of Arabidopsis thaliana PHYC ${ }^{33}$ ) showed a significant 191 phenotypic difference for $\operatorname{DTT}_{\mathrm{HN}}\left(\mathrm{P}=1.2 \times 10^{-7}\right.$; Fig. 2D) and the specific plasticity 192 measures, calculated as the difference between $\mathrm{HN}$ to the other sites (e.g. $\mathrm{DTT}_{\mathrm{HN}-\mathrm{BJ}} ; \mathrm{P}=5.3 \mathrm{x}$ $19310^{-8}$; Fig. 2E), but had no effect at the remaining DTT mean and plasticity measurements (Fig. 194 2C-E), indicating changes in the magnitude of genetic effects contributed to the variation of 
195 DTT plasticity. In contrast, QTL14 (chromosome 9: 35,126,793 bp, Fig. 2A-C, F, 508.5 kb upstream of CONZ1, GRMZM2G405368, a homology of Arabidopsis thaliana $C O^{34}$ ), was exclusively detected for several DTT plasticity measurements but not for any of the DTT $_{\text {mean }}$ and $\mathrm{DTT}_{\mathrm{BLUP}}$. The genetic effects of QTL14, however, changed direction from positive $\left(\mathrm{DTT}_{\mathrm{HN}}\right.$, Additive effect $=0.6 \pm 0.2$ days; $\mathrm{P}=1.7 \times 10^{-3} ;$ Fig. $\left.2 \mathrm{~F}\right)$ to negative $\left(\mathrm{DTT}_{\mathrm{HN}}\right.$, Additive effect $=-0.5 \pm 0.2$ days; $\mathrm{P}=7.3 \times 10^{-3}$; Fig. $2 \mathrm{~F}$ ), leading to significant association with specific plasticity, $\mathrm{DTT}_{\mathrm{HN}-\mathrm{BJ}}$ (Additive effect $=1.1 \pm 0.2$ days; $\mathrm{P}=6.3 \times 10^{-12}$; Fig. 2D) and overall plasticity, $\mathrm{DTT}_{\mathrm{pc} 2}\left(\mathrm{P}=4.1 \times 10^{-9}\right)$, The detection of such loci highlighted the increased power by analysing plasticity measurements.

204 Altogether, these results indicated that changes in magnitude and/or signs of genetic effects across sites caused variation in plasticity, which could be detected by GWAS on SP and OP measurements. The changing genetic effects highlighted the role of QTL by environment interaction in the variation of complex trait mean and plasticity.

A

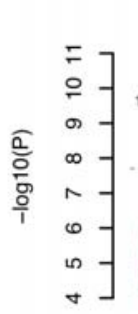

C

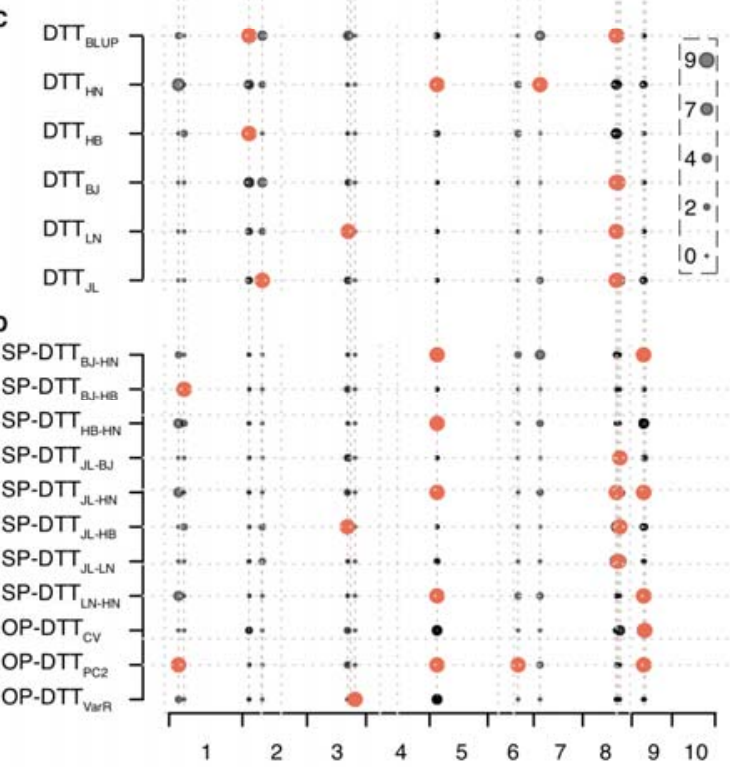

B

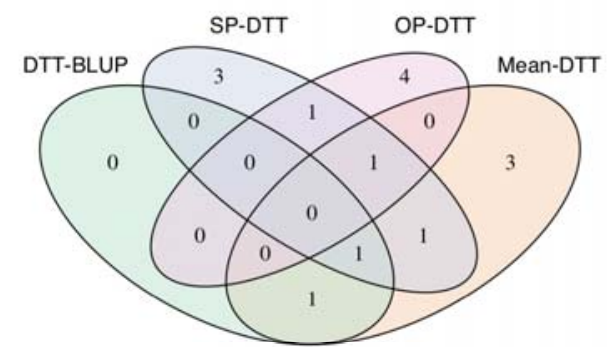

E

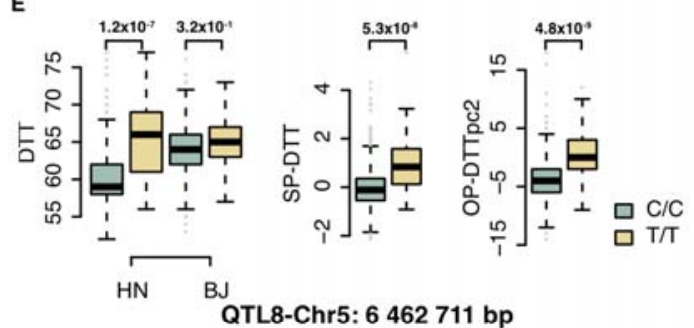

$\mathbf{F}$

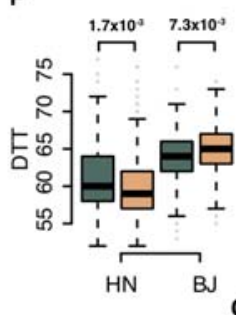

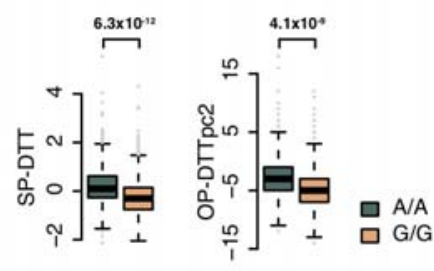

QTL14-Chr9: 35126793 bp

Fig. 2 Summary of the QTLs associated with the mean and plasticity measures for days 
213 effective number of independent SNPs; Materials and Methods), and the vertical dashed 214 black lines indicate the position of detected QTLs, labelled from 1 to 15 . B) Venn diagram 215 illustrating the overlap of QTLs detected for the 4 types of DTT measurements. C) QTLs 216 associated with the DTT means measured at five sites and the DTT BLUP $_{\text {(y-axis). Each dot }}$ 217 represents a SNP and the size of the dot is proportional to its - $\log 10 \mathrm{p}$ value as indicated in 218 the legend on the right. Loci with p-value passed genome wide significance threshold were 219 coloured in tomato. D) QTLs associated with the DTT plasticity measurements (labelled in

the y-axis). E) and F) Genotype-to-phenotype maps, highlighting the increased power to detect additional loci by analysing plasticity measurements, for DTT $_{\mathrm{HN}}, \mathrm{DTT}_{\mathrm{BJ}}, \mathrm{DTT}_{\mathrm{HN}-\mathrm{BJ}}$, and DTT $_{\mathrm{pc} 2}$ at two QTLs, one at chromosome 5: 6,462,711 bp and a second one at chromosome 9: 35,126,793 bp.

Loci associated with the variation of remaining traits- A complex genetic architecture involving multiallelic, pleiotropy, and genotype by environment interaction underlay maize complex trait variation

228 For the 23 traits, we identified 109 QTLs for the 4 types of measurements (Fig. 3A; Figure S3; Table S3, S4, S6), which overlapped partially, with $1.8 \%, 34.9 \%, 19.3 \%$, and $21.1 \%$ of the QTLs being unique to BLUP, SP, OP and Mean measurements, respectively (Fig. 3B). As has been illustrated in the previous section, QTLs associated with SP measurements likely changed their genetic effects in sign or direction (Fig. 2E, F). This was supported by testing the interaction between the detected QTLs and the five sites, where $80.0 \%$ of the QTLs were found to be significantly interacting with the sites (Table S7; Materials and methods). This demonstrated the dynamic genetic effects of mean QTLs across sites and highlighted partial overlap for QTLs regulating mean and plasticity as reported in the previous studies ${ }^{24,25,27}$.

237 One QTL, spanning $540 \mathrm{~kb}$ from chromosome 5: 6,382,800 bp to 6,923,292 bp, involved 7 238 statistically independent SNPs (Cluster 1 in Fig. 3A, C, QTL 8 in Fig. 2A) and was detected 239 for multiple trait means and plasticity measures at HN. A detailed exploration showed that 240 multiple haplotypes were underlying this region with each of the 7 SNPs tagging unique 241 haplotype (Figure S4), suggesting that the 24 founders carried different functional variants.

242 Moreover, each of the 7 SNPs was simultaneously associated with multiple trait means at HN, 243 including flowering time $\left(\mathrm{DTT}_{\mathrm{HN}}\right.$ and $\left.\mathrm{DTA}_{\mathrm{HN}}\right)$, plant architecture trait (the ear leaf width, 244 ELW $_{\mathrm{HN}}$ ), and multiple SP measurements (SP-DTT, SP-DTA, SP-DTS, SP-PH, SP-EW) at 245 genome wide significance (Fig. 3C, D). Moderate association to the mean and plasticity 246 measurements for yield and plant architecture traits were also found at a relaxed significance 247 threshold $\left(\mathrm{P}=6.0 \times 10^{-6}\right.$ for $\mathrm{EW}_{\mathrm{HN}}$ and $\mathrm{P}=5.1 \times 10^{-5}$ for $\mathrm{PH}_{\mathrm{HN}}$; Fig. 3D), indicating this 248 region was highly pleiotropic. Notably, the genetic effect of this QTL was unique to HN for 
249 all the associated traits, where the "TT" genotype increased DTT, DTA, DTS and "CC" 250 decreased ED, EW, ELW, and PH at HN but not at other sites, likely due to interaction with 251 temperature (Especially TemD) rather than DayL (Fig. 3E; Figure S5), providing an ideal 252 candidate for targeted breeding application at $\mathrm{HN}$.

A

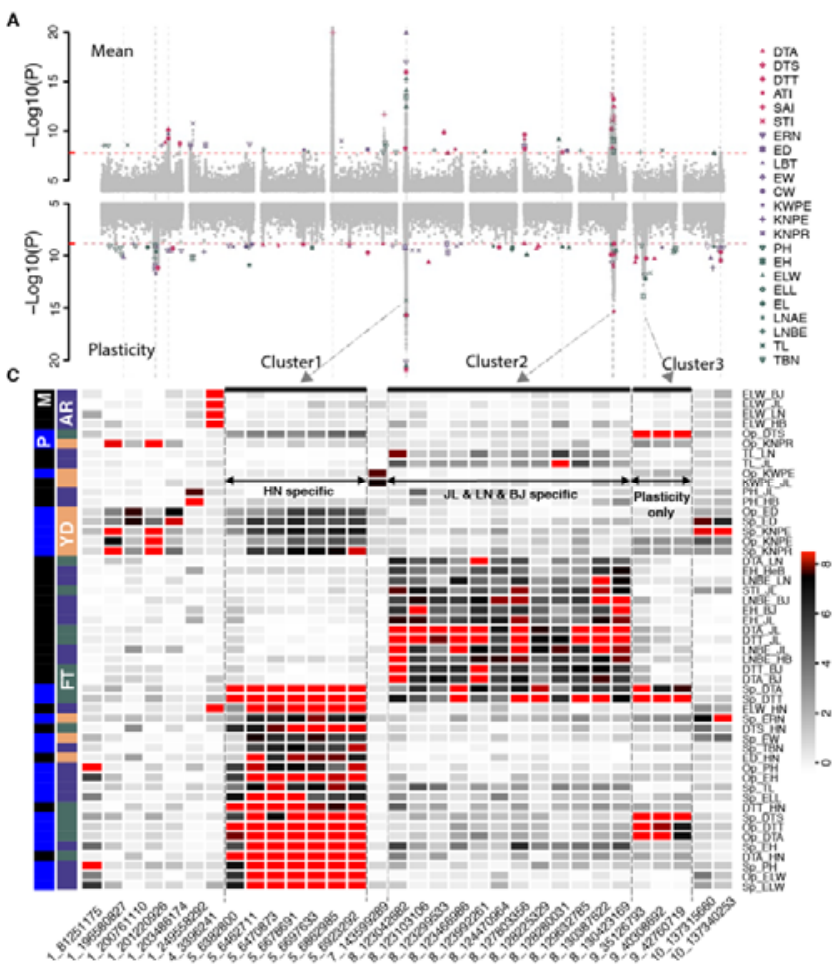

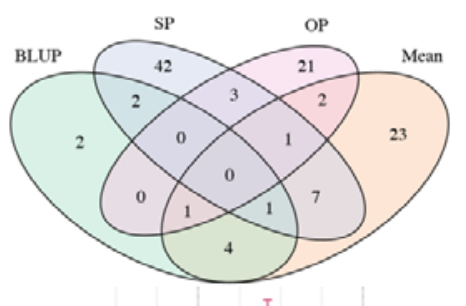

D

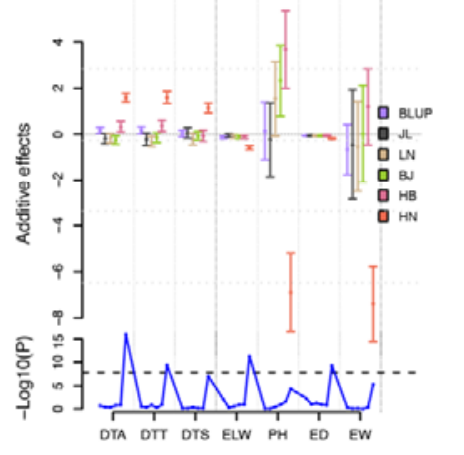

E

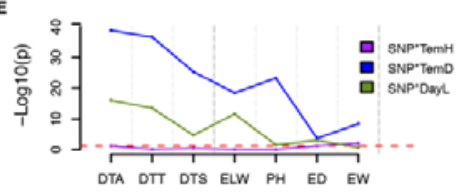

Fig.3 Association results of both mean and plasticity for all the 23 traits. A) Manhattan measurements. The red horizontal dashed lines indicate the Bonferroni-corrected genomewide significance threshold. The vertical dashed grey lines highlight the site of 32 SNPs associated with more than 2 measurements. B) Venn diagram illustrating the overlap of QTLs detected for the 4 types of measurements. C) A heatmap illustrating the p values of the 32 SNPs detected for more than 2 measurements (Here, SNPs were used instead of QTLs, as one QTL sometimes includes multiple statistically independent SNPs that are physically close to each other). Each cell represents the -Log10 (p values) of a particular SNP (x-axis) associated with a specific trait (y-axis on the right). The outer index on the left side marks the mean (M, in black) or plasticity ( $\mathrm{P}$, in blue) of the traits. The inner index marks the corresponding trait types: plant architecture (AR; in purple), flowering time (FT; in olive-green), and yield (YD; in orange). For each trait, only the lowest p-values were indicated for either specific plasticity (SP) or overall plasticity (OP), labelled as SP-trait or OP-trait. D) The Additive effects varied across sites exampled for cluster 1 (chromosome 5:6 $462711 \mathrm{bp}$ ) on multiple traits. The traits were separated by the dashed vertical lines and labelled in x-axis, and for each trait the measurements for BLUP, individual sites were ordered (from left to right) as indicated in the colour legend (from top to bottom). Median and standard error were shown with the middle point and error bars. The corresponding GWAS p values were illustrated in the lower panel. the 3 environmental factors. 
276 A second cluster, spanning 7.4 Mb from chromosome 8: 123,042,682 bp to 130,423,169 bp,

277 showed both allelic heterogeneity and pleiotropic effect on multiple flowering and plant

278 architecture traits (Cluster 2 in Fig. 3A, C; Figure S6). However, their genetic effects were

279 unique to the three northern sites (JL, LN, and BJ; Fig. 3C), except for LNBE $\mathrm{HB}_{\text {. Compared }}$

280 with cluster 1 , whose effects were unique to $\mathrm{HN}$, such regional effects on multiple northern

281 sites may have led to the detection of this QTL for multiple sites BLUP measurements.

282 A third cluster (Cluster 3 in Fig. 3A, C) was found contributing exclusively to the variation of 283 plasticity measurements for all the flowering time traits (DTT, DTA, and DTS) due to the 284 change of additive effects from negative to positive (Fig. 2F).

285 Altogether, these results illustrated a complex genetic architecture involving multiallelic, 286 pleiotropy, and genotype by environment interaction underlying maize complex trait 287 variation. The detection of QTL unique to HN and the three northern sites demonstrated a 288 variable genetic architecture of maize complex traits across sites possibly due to clinal 289 variation in QTL effects.

\section{The possible molecular basis of phenotype plasticity}

292 Previously, we linked ZmTPS14.1 (GRMZM2G068943, chromosome 8: 123,129,008 bp to $293123,140,283 \mathrm{bp}$ ) to variation of flowering time mean ${ }^{35}$, which was located inside the QTL on 294 chromosome 8 (cluster 2 in Fig. 3, chromosome 8: 123,042,682 bp to 130,423,169 bp). Here, 295 this QTL was simultaneously associated with mean and plasticity variation of multiple traits, 296 including DTT, DTA, DTS, ATI, STI, SAI, LNAE, and LNBE at genome-wide (P =1.53 x $\left.29710^{-8}\right)$ or suggestive significance threshold $\left(\mathrm{P}=1.00 \times 10^{-5}\right)$ and the tagging SNPs were 298 interacting with all 3 environmental factors, suggesting a general contribution from QTL by 299 environmental factor interaction to variation in phenotype plasticity.

300 To experimentally validate and evaluate the plasticity effects of ZmTPS14.1, we planted the 301 knock-out lines of ZmTPS14.1 obtained in a previous sudty ${ }^{35}$ in Jilin (JL, North China, N $43^{\circ}$ $30230^{\prime}$, E $124^{\circ} 49^{\prime}$ ) and Hainan (HaiN, South China, N $18^{\circ} 34^{\prime}$, E $108^{\circ} 43^{\prime}$ ) and compared the 303 measured flowering time phenotypes. In consistent with the association results, the female 304 flowering time (DTS) of knock-out lines was earlier in HaiN but not significantly changed in 305 JL compared to wildtype lines (Fig. 4A; Figure S7A; Table S8). To further explore the 
306 underlying molecular basis, we analysed an in-house time-course transcriptome dataset 307 generated from reference accession B73 under long-day and short-day conditions (Fig. 4B).

308 The expression of ZmTPS14.1 under both day-length conditions changed in the same 309 direction along the time course (Fig. 4B), suggesting there was no day-length dependent 310 expression response for ZmTPS14.1.

311 As has been proposed that plastic response may involve developmental switch genes ${ }^{36}$, we 312 explored whether plastic effects of genes at the center of the regulatory pathway were 313 mediated or interacted with downstream genes. Therefore, we evaluated the expression of 314 candidates downstream of ZmTPS14.1. ZmTPS14.1 encodes Trehalose-6-phosphate synthase 315 (TPS), which converts glucose-6-phosphate into Trehalose-6-phosphate (T6P), regulating 316 vegetative development and flowering by miR156/SPL pathway ${ }^{37}$. The expression of 317 ZmSPL6 (GRMZM5G878561), an SPL family member downstream of ZmTPS14.1, showed a 318 significant expression pattern difference in response to long/short day length (Fig. 4B). 319 Meanwhile, the knock-out lines of ZmSPL6 showed earlier female flowering in JL but no 320 significant change in HaiN compared to wildtype lines (Fig. 4A; Figure S7B; Table S8), 321 suggesting day length was an important factor for the plastic effect of ZmSPL6. Thus, we 322 proposed a compensation mechanism from ZmSPL6 to ZmTPS14.1 in DTS plasticity (Fig. $3234 \mathrm{C})$. In the long-day condition, the continuous expression increase of ZmSPL6 could make up 324 for the knockout effect of ZmTPS14.1, resulting in no phenotypic difference between the 325 knock-out lines of ZmTPS14.1 and wildtype (Fig. 4A). But no such compensation appeared 326 in the short-day condition, thus we observed the phenotype difference between knockout and 327 wildtype lines of ZmTPS14.1 in the short-day condition (Fig. 4A, C). This compensation 328 mechanism was also reflected in the CUBIC population (Fig. 4D). In the long-day condition 329 (JL), ZmTPS14.1 (chromosome 8: 123,138,468 bp) showed significant association ( $\mathrm{P}=1.5 \mathrm{x}$ $33010^{-2}$ ) with DTS in the TT allele background of ZmSPL6 (-), but not significant in GG allele 331 background of ZmSPL6 (+). And in the short-day condition (HN), the significant association 332 between ZmTPS14.1 and DTS was detected in both ZmSPL6 (-) $\left(\mathrm{P}=8.9 \times 10^{-5}\right)$ or ZmSPL6 $333(+)\left(\mathrm{P}=5.0 \times 10^{-3}\right)$ backgrounds (Fig. 4D). 


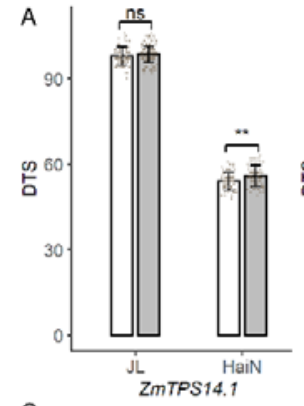

C

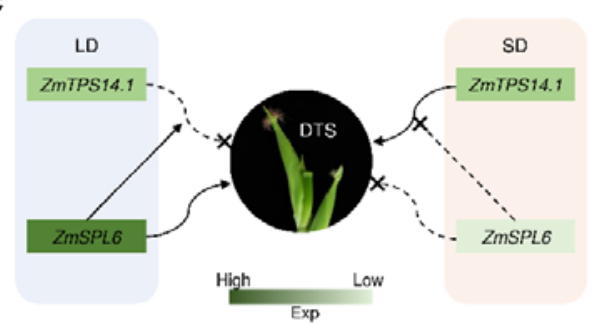

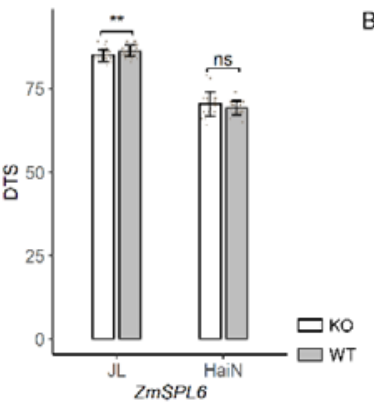

D

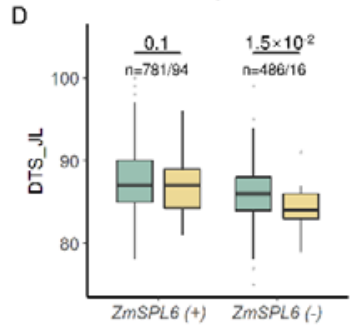

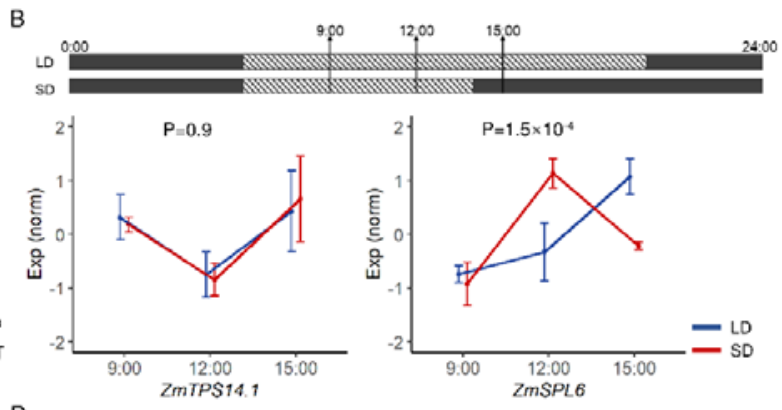

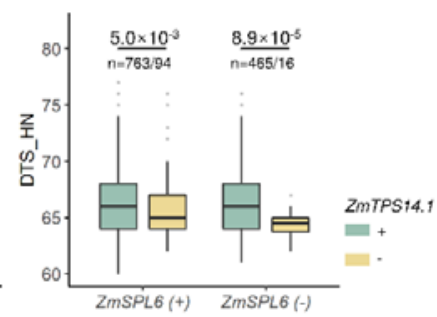

335

336

337

338

339

340

341

342

343

344

345

346

347

348

349

350

351

352

353

354

355

356

357

358

359

360

361

362

363

Fig. 4 The interaction between ZmTPS14.1 and ZmSPL6 reveals the genetic basis of phenotype plasticity of flowering time. A) Phenotype (DTS; days to silking) of knock-out lines and wild type of ZmTPS14.1 and ZmSPL6 at two field plantations, one plantation at JL represents Jilin $\left(\mathrm{N} 43^{\circ} 30^{\prime}\right.$, E $\left.124^{\circ} 49^{\prime}\right)$, and another one at HaiN represents Hainan $\left(\mathrm{N} 18^{\circ}\right.$ $\left.34^{\prime}, \mathrm{E} 108^{\circ} 43^{\prime}\right)$. Error bars represent standard deviation. ** indicate $\mathrm{P}$ values $<0.01$ by Student's t-test. "ns" means no significance. B) The sampling diagram of the time-course experiment in B73 under long-day (LD) and short-day (SD) conditions. The black area represents dark time and the dotted-line area represents light time. Leaf tissues were harvested at three time points (9:00, 3 hours of light; 12:00, 6 hours of light; 15:00, 9 hours of light/ 1 hour of dark). The expression pattern of ZmTPS14.1 and ZmSPL6 at three time points under the long-day condition (LD, blue) and the short-day condition (SD, red) were shown. The $y$-axis represents gene expression, which was obtained from standardization of raw reads counts then z-score normalization. Error bars represent standard error. C) The proposed compensation interaction model between ZmSPL6 and ZmTPS14.1. ZmSPL6 expressed highly in the long-day condition which could promote female flowering but its expression suppressed in the short-day condition (SD) showed no effect for flowering. And the knockout lines of ZmTPS14.1 showed the flowering time difference in the short-day condition, but not in the long-day condition because of the compensation effect of ZmSPL6. D) The phenotype (DTS in JL and HN) comparison between two alleles of ZmTPS14.1 (chromosome 8: $123,138,468 \mathrm{bp} ; \mathrm{C} / \mathrm{C}$ genotype $\rightarrow++$; T/T genotype $\rightarrow-$ ) in the different allele background of ZmSPL6 in LD (JL) and SD (HN) conditions (chromosome 3: 159,420,596 bp; G/G genotype $\rightarrow+$; T/T genotype $\rightarrow-$ ). P-values were obtained by Student's t-test.

Accounting for dynamics in genetic architecture improved complex traits prediction across environments

We evaluated the potential of integrating genetic diversity, environmental variation, and their interaction in complex trait prediction by jointly modelling genotype, environment, and their interaction (referred to as GEAI model, Materials and Methods). Two cross-validation schemes were considered. In the first case, we explored the predictability on untested lines at 
364 any of the five sites by using all the lines phenotyped at the five sites using 5-fold cross-

365 validation. Compared with the GBLUP, with a universal prediction for all sites, our model 366 not only provided site-specific predictions but also increased prediction accuracy for a 367 majority of traits and sites (83.0\% of all traits and sites; Table S9, Materials and methods). 368 The averaged prediction accuracy for DTT, PH and EW increased by 5.3\%, 1.2\%, and 1.8\%, 369 respectively, and the increase in prediction accuracy was more pronounced at HN (increased 370 by $15.5 \%, 3.8 \%$, and $4.4 \%$ for DTT, PH, and EW, respectively, Fig. 5A-C).

371 In the second case, we explored a serial of more challenging designs, in which only a core set 372 of lines $(10 \%-70 \%)$ were phenotyped across five sites and the interest was to predict the 373 performance of unphenotyped lines at each site. It was very encouraging to see that our GEAI 374 model showed higher accuracy for almost all the traits and sites. For example, at 10\% overlap, 375 our GEAI model outperformed GBLUP predictions $\left(\mathrm{P}=4.0 \times 10^{-3}\right)$ by $3.2 \%$ on average and 376 increased the prediction accuracy at four out of five sites by $1.0 \%-12.7 \%$ for DTT, and the 377 averaged accuracy was increased to $4.6 \%$. At $70 \%$ overlap, the increase in accuracy at each 378 site was larger than at $10 \%$ overlap (1.5\%-13.5\%; Fig. 5D-I). As the number of lines 379 phenotyped at all sites increased from $10 \%$ to $70 \%$, both the averaged accuracies and site380 specific accuracies increased (Figure S8). Overall, our study highlighted the potential of 381 intergrading QTL by environment interaction in understanding complex traits variation and 382 predictions. 

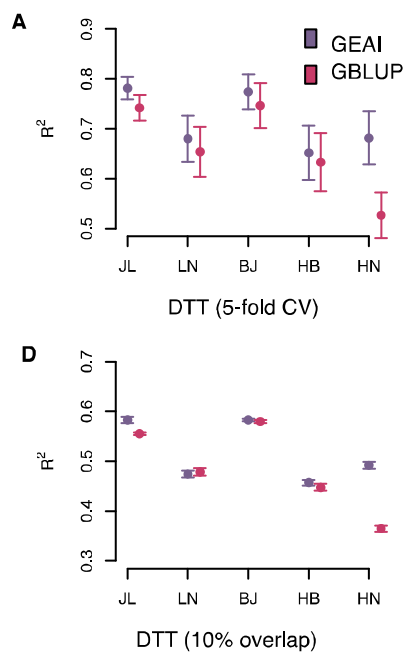

G

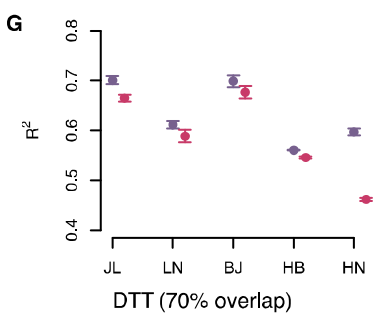

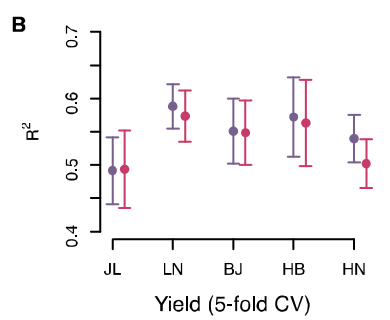
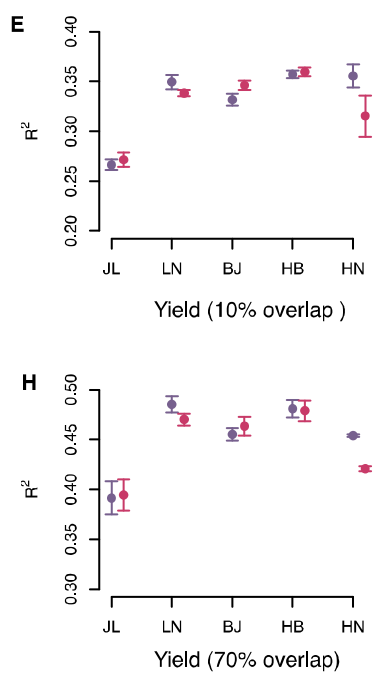
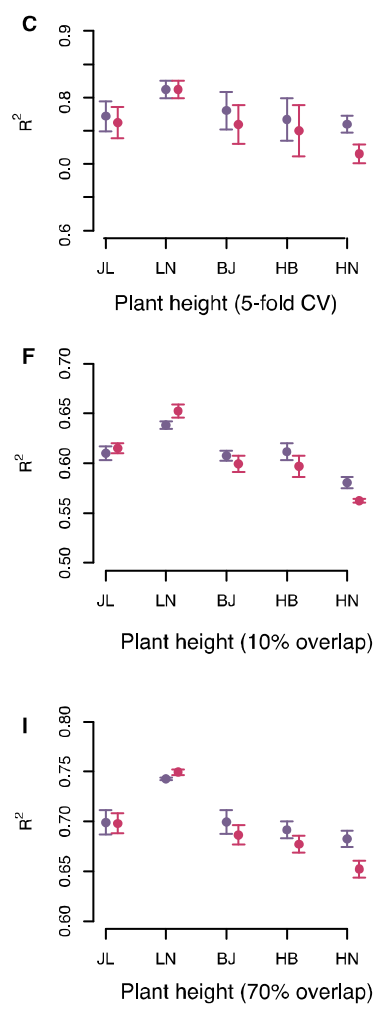

383

Fig. 5 Performance of GEAI model in site-specific complex trait prediction. A) Predictability of untested lines at any of the five sites by using all the lines phenotyped across the five sites as training data for A) DTT, B) EW, and C) PH. Prediction accuracy for untested lines at any of the five sites for D) DTT, E) EW, and F) PH when $10 \%$ of the lines were phenotyped at all five sites and remaining lines were only phenotyped at one of the five sites. Prediction accuracy for G) DTT, H) EW, and I) PH when $70 \%$ of the lines were phenotyped at all five sites and the remaining lines were only phenotyped at one of the five sites.

\section{Discussion}

Here, by surveying the performance of a genetically diversified population across China's summer maize major production zone, we were able to quantify contributions from specific environmental factors to the variation of 23 complex traits, detect plastic QTLs, and provide site-specific complex traits prediction model with higher accuracy.

Contribution from environmental factors to maize complex traits mean variation and plasticity variation

Plants time their vegetative and reproductive growth in response to changes in seasonal cues, such as winter temperatures (vernalization) and day length (photoperiod) ${ }^{38}$. Although many 
402 studies have emphasized the importance of photoperiod to flowering time regulation, the 403 temperature is a key determinant of flowering time ${ }^{39-41}$, and significantly stronger

404 correlations between seasonal transcriptome and temperature than those with day length ${ }^{42}$ 405 were reported. In consistent with these reports, we found that TemH had a considerable high 406 contribution to the across-environment variation of flowering traits, while very little 407 contribution from TemD, DayL, G-by-TemH, or G-by-DayL were found. In contrast, yield 408 traits were influenced by a combination of TemH, TemD, DayL, and their interactions with 409 genotype. A possible explanation is that both photosynthesis and respiration losses, mainly 410 determining the crop yields, are sensitive to temperature and day length ${ }^{43}$, and previous 411 studies have shown that temperature and day length could also affect days to maturity, rate of 412 yield accumulation, and harvest index ${ }^{44}$.

413 Unfortunately, soil conditions, such as $\mathrm{pH}$, soil temperature, water, and nutation content were 414 not available in our study, limiting our ability to provide broader insight into the impact of 415 specific environmental factors on complex trait variation. The genotype to filed (G2F) Maize 416 project $^{45}$, one of the ongoing efforts aiming at compensatively surveying the environmental 417 factors and performance of diversified population across a large number of field plantations, 418 would be of great importance to characterise the role of specific soil factors on complex traits 419 variation.

420 Here, we quantified phenotype plasticity as a response to changes between particular 421 environment sites and across all five sites, resulting in multiple plasticity measures for the 422 same genotype. Despite a high overall correlation among these plasticity measurements, 423 different QTLs were detected, indicating that these measures captured different aspects of 424 plasticity with complementary information. Such differences in quantifying phenotype 425 plasticity may be highly relevant in applications where the testing site and targeted site are 426 clearly defined. In particular, when the mechanism of environmental factors interacting with 427 the plastic QTL is known, an accurate prediction could be made on germplasm performances 428 under various deployment environments at the GenBank level, facilitating precise breeding 429 designs in the future.

430 Among the 23 traits, yield traits were more plastic than other traits and involved larger 431 contributions from both temperature and day length, as well as a larger proportion of G-by-E 432 interaction. A similar result has been reported in D'Andrea et al (2013) ${ }^{46}$. A possible 433 explanation could be that yield traits were the results of combined effects from vegetative and 434 reproductive growth with demonstrated contribution from both temperature and 
435 photoperiod ${ }^{43,44}$ that likely to be equally important, while flowering time was predominantly

436 regulated by temperature ${ }^{42}$ with a relatively smaller contribution from photoperiod. Future

437 studies are required to explore how differences in genetic architecture among traits cause

438 such differences in phenotypic plasticity.

\section{The genetic architectures underlying trait mean and plasticity}

440 In consistent with previous studies ${ }^{25,47}$, we found partial overlaps between QTLs associated 441 with trait mean and plasticity. However, our interpretation is that when treating phenotype 442 plasticity as a measure of change for one polygenic trait across environments, such overlap is 443 expected. Besides this, we also expect that i) plasticity is polygenic as a result of the 444 polygenic architecture for the trait itself at different environments, ii) the degree of overlap 445 between QTLs underlying trait mean and plasticity may vary across studies due to detection 446 power, and iii) QTLs with altered genetic effect among environments are more likely to 447 impact the variability of plasticity. Taking DTT as an example, we detected 7 loci for DTT 448 mean and 9 loci for DTT plasticity with four loci overlapping. The magnitude change 449 (chromosome 5: 6,462,711 bp) or the sign change (chromosome 9: 35,126,793 bp) of QTL 450 effects resulted in variability in DTT plasticity, providing support for the allelic sensitivity 451 model $^{48}$. Even though we did not detect the chromosome 9 QTL in DTT mean scan at a 452 genome-wide significance, a moderate association was found at a lower significance 453 threshold. In line with this, when aggregating the allelic effects of mean or plasticity QTLs 454 not detectable at genome-wide significance, we found, as a group, they were significantly 455 contributing to the variation of both mean and plasticity measurements (Figure S9, Table 456 S10). Given the polygenic and dynamic genetic architecture of trait mean across 457 environments reported here and in previous research ${ }^{30,49}$, there might be a tighter connection 458 between the genetic regulation of trait mean and plastic than we have previously 459 acknowledged.

460 Plasticity QTL may have been subjected to directional selection during the breeding 461 program

462 Previous studies showed that the highly selected region during maize adaptation to temperate 463 climate explained less G-by-E variation than the selected region ${ }^{12}$ and the allele frequency of 464 plasticity QTLs were changed between temperate and tropical lines ${ }^{27}$, suggesting that 465 directional selection may have shaped their genetic diversity. Here, we explored whether the 46693 plasticity QTLs were selected during intense artificial selection by evaluating their allele 467 frequency changes using two collections of breeding materials, one collection from China 
468 that has predominantly been deployed in the 1960s, 1980s, and early 2000s, and a second 469 collection from US before and after $2003^{50}$. We found that the allelic frequencies of $42(45 \%)$ 470 plasticity QTLs consistently changed from 1960s to 1980s and from 1980s to early 2000 in 471 Chinese collection, and before and after 2003 in US collection (Figure S10). Such an 472 agreement indicates that it is likely that these plastic QTLs were subjected to selection rather 473 than genetic drift. However, many plastic QTLs, found here or in earlier researches ${ }^{24,25}$, were 474 also contributing to the trait mean, further studies would be required to explore whether such 475 changes are results of directional selection or simply consequences of selection on the trait 476 mean that is correlated with the plasticity.

\section{Fine mapping the QTLs and the molecular basis of variation in phenotype plasticity}

478 We found that a few QTL peaks, such as the QTL on chromosome 5: 6-7 Mb (Fig. 3A; 479 Figure S4) and chromosome 8: 123-130 Mb (Fig. 3A; Figure S6) simultaneously associated 480 with multiple traits means and plasticity measurements, possibly being a consequence of 481 extended linkage disequilibrium (LD). Fine mapping the causal variants underlying each trait 482 mean and plasticity QTL and distinguishing whether these signals were tagging one common 483 signal simultaneously associated with multiple trait means and plasticity measures or they 484 were multiple variants each associated with one measure but in tight LD with each other is a 485 daunting task. Even though detailed analysis (Supplementary note) showed that a large 486 proportion of the SNPs were tagging the same causal variants (Figure S4, S6), there seemed 487 to be multiple independent association signals underlying the same QTL (Figure S4, S6) for a 488 few mean or plasticity measures. For example, a detailed exploration on the chromosome 5 489 QTL showed that multiple statistical independent SNPs were tagging different combinations 490 of multiple functional haplotypes (Figure S4), illustrating a complex genetic architecture 491 involving allelic heterogeneity, multi-allelic, pleiotropy, and genotype by environment 492 interaction at the same time. To pinpoint the causal genes in presence of such complexity, we 493 applied gene-based test ${ }^{51}$ aggregating summary statistics on SNPs up/down stream of the 494 annotated protein-coding genes, and detected 300 genes (Table S11), among which 24\% were 495 simultaneously associated with both mean and plasticity measurements (106 for mean, 122 496 for plasticity, 72 for both). Among these genes, the maize FT gene ZCN8 was detected in 497 both means and plasticity of flowering traits, while ZCN18 was only associated with STI 498 plasticity $^{52}$. A benzoxazinone synthesis gene cluster including bxl/2/3/8 on chromosome 4 499 was detected with association to the mean of ELW. Similar conditional effects also had been 500 found in mutant and overexpression of multiple flowering genes in Arabidopsis, such as 
501 PRR3 in circadian clock $^{53}$, PIF4 in ambient temperature pathway ${ }^{54}$, and $H X K 1$ in sugar

502 pathway $^{55}$. Although future experimental validations are required to validate the biological

503 mechanism undying such variation, the validation of two candidate genes in our study

504 suggests that the effect of genes on complex traits may in general be context-dependent.

\section{Conclusion}

506 In summary, we showed that the genetic architectures of maize agronomic traits were

507 dynamic across China's major summer maize production zone with the genetic effects of

508 many QTLs being either local or regional due to interaction with environmental factors,

509 leading to changes in additive genetic variance, narrow sense heritability and variation in

510 phenotype plasticity. The dynamic allelic effects of plasticity QTLs enable us to develop a

511 GEAI complex trait prediction model with site-specific predictions and higher accuracy,

512 opening a new possibility for future crop improvement. Our study thus provided novel

513 insights into the dynamic genetic architectures of agronomic traits in response to changing

514 climate and provided a GEAI model with site-specific prediction, paving a practical route to

515 precision agriculture.

516

\section{Materials and methods}

\section{Experimental design}

519 We developed a Complete-diallel plus Unbalanced Breeding-derived Inter-Cross (CUBIC) 520 population of 1404 maize inbred lines and surveyed their performance for 23 agronomic traits 521 at five sites in China's major maize production zone with longitudinal variation from E $114^{\circ}$ $52201^{\prime}$ at Henan $(\mathrm{HN})$ to $\mathrm{E} 125^{\circ} 18^{\prime}$ at Jilin (JL) and latitudinal variation from $\mathrm{N} 43^{\circ} 42^{\prime}$ at $\mathrm{HN}$ to $523 \mathrm{~N} 35^{\circ} 27^{\prime}$ at $\mathrm{HN}$. A detailed description of the development of this population was available 524 in Liu et $\mathrm{al}^{29}$. Briefly, these inbred lines were derived from 24 elites representing 4 divergent 525 heterotic groups with cycles of random mating, selection, and inbreeding ${ }^{29}$. In 2014, all 526 inbred lines, each with five replicates, were planted at five sites, including Jilin Province (JL, $527 \mathrm{~N} 43^{\circ} 42^{\prime}$, E $125^{\circ} 18^{\prime}$ ), Liaoning Province (LN; N 42 $03^{\prime}$, E $123^{\circ} 33^{\prime}$ ), Beijing (BJ; N 40 $52810^{\prime}$, E $\left.116^{\circ} 21^{\prime}\right)$, Hebei Province (HB, N $38^{\circ} 39^{\prime}$, E $\left.115^{\circ} 51^{\prime}\right)$ and Henan Province (HN; N $35^{\circ}$ $52927^{\prime}, \mathrm{E} 114^{\circ} 01^{\prime}$ ). Twenty-three agronomic traits, including 6 phonology traits, 8 plant 530 architecture traits, and 9 yield traits were phenotypically evaluated. Except for six flowering 531 traits that were scored as the median values of replicated lines, all the remaining traits were 532 scored as the means among replicates (Table S12). Three environmental variables, including 
533 daily highest temperature $(\mathrm{TemH})$, daily temperature difference (TemD), and day length

534 (DayL), were extracted from local weather stations

535 (http://data.sheshiyuanyi.com/WeatherData/). All the 1404 lines were re-sequenced and 536 called genotypes were available for download from Liu et $\mathrm{al}^{29}$. Totally $6.6 \mathrm{M}$ SNPs with $537 \mathrm{MAF}>0.03$ and $\mathrm{LD}=<0.9$ in $100 \mathrm{~kb}$ sliding window were retained for downstream analysis.

538 Partition the phenotypic variance into contributions from genotype, environment factors, 539 and their interactions

540 The phenotypic variance was partitioned into contributions from genotype (G), genotype-by541 environment (G-by-E), and residual (environment; E) by fitting the following model:

$542 \overline{\mathrm{y}}_{\mathrm{ij}}=\mathrm{u}+i d_{i}+\mathrm{TemH}_{\mathrm{j}}+\mathrm{TemD}_{\mathrm{j}}+\mathrm{DayL}_{\mathrm{j}}+i d_{i} * \mathrm{TemH}_{j}+i d_{i} * \mathrm{TemD}_{j}+i d_{i} * \mathrm{DayL}_{j}+e_{i j}(1)$

543 This model was fitted for each of the 23 traits one at a time. $\bar{y}_{i j}$ is the trait mean/median of 544 individual $\mathrm{i}(\mathrm{i}=1 \ldots \mathrm{n}, \mathrm{n}=1404$ is the number of individuals) at site $\mathrm{j}(\mathrm{j}=1 \ldots \mathrm{q} ; \mathrm{q}=5$, 545 corresponds to the number of sites); $\mathrm{id}_{\mathrm{i}}$ is the line $\mathrm{id}$ (genotype) coded as factor; $546 \mathrm{TemH}_{\mathrm{j}}, \mathrm{TemD}_{j}$ and $\mathrm{DayL}_{\mathrm{j}}$ are three environmental variables representing the daily highest 547 temperature, daily temperature difference, and day length at site $\mathrm{j}$, respectively. These 548 environmental factors were coded as numeric, assuming a linear relationship with the 549 phenotypic measurements. $i d_{i} * \mathrm{TemH}_{\mathrm{j}}, i d_{i} * \mathrm{TemD}_{\mathrm{j}}$, and $i d_{i} * \mathrm{DayL}_{\mathrm{j}}$ are the interaction terms 550 (G-by-E) between a particular line (genotype) and the corresponding environmental factors 551 (environment). The relative contributions to the total phenotypic variance from $\mathrm{G}$ and G-by-E 552 were estimated by their respective sum of squares (Sum of Square for id is calculated as $553 \sum_{1}^{p}\left(i d_{i}-\overline{l d}\right)^{2}$ and Sum of Square for the interaction terms id*E are calculated as $\sum_{1}^{n}\left(i d_{i} *\right.$ $\left.554 \mathrm{E}_{j}-\overline{\imath d_{\imath} * \mathrm{E}_{j}}\right)^{2}$ ), where E stands for TemH, TemD, and DayL.

555 Estimating the narrow-sense heritability, additive variance, and genetic correlations

556 A linear model was used to estimate the narrow-sense heritability for all the 23 traits 557 measured at each of the five sites.

$558 \bar{Y}=\mu+\mathrm{Zu}+\mathrm{e}(2)$

559 Here, $\bar{Y}$ is a vector of trait mean/median of each individual (genotype) at each tested site. $e$ is 560 the normally distributed residual. $\mu$ is a column vector of 1's to represent the population 561 mean, and $\mathrm{u}$ is a random effect vector of the breeding values for the 1404 individuals. $\mathrm{Z}$ is the 562 corresponding design matrix obtained from a Cholesky decomposition of the kinship matrix $563 \mathrm{G}$, estimated using the genome-wide markers using $\mathrm{GCTA}^{56}$. The $\mathrm{Z}$ matrix satisfies $\mathrm{ZZ}^{\prime}=\mathrm{G}$, 
564 therefore, that is normally distributed $\left(\mathrm{u} \sim \mathrm{N}\left(0, I \sigma_{g}^{2}\right)\right)$. e is the residual variance with e $\sim \mathrm{N}(0$,

$\left.565 I \sigma_{e}^{2}\right)$. The narrow-sense heritability of fitted phenotype was calculated as the intraclass

566 correlation $h^{2}=\sigma_{g}^{2} /\left(\sigma_{g}^{2}+\sigma_{e}^{2}\right)$. AI-REML implemented in GCTA was used to obtain these

567 estimates $^{56}$. The additive genetic variance was then estimated as the variance of $\mathrm{Y}\left(\operatorname{Var}_{\mathrm{Y}}\right)$

568 times $h^{2}$.

569 Similarly, a bivariant mixed model was fitted to obtain estimates of the genetic correlation 570 between measurements obtained from two individual sites. Ten models were thus fitted to 571 obtain all the pairwise genetic correlations among five sites. $\bar{Y}, \mu$ and $\mathrm{u}$ from the model (2)

572 were updated to an $n \times 2$ matrix, with $n$ being the number of individuals and each column

573 vector representing measurement obtained from a particular site. This model was fitted using 574 the reml-bivar module ${ }^{57}$ implemented in the GCTA software ${ }^{56}$ and details of this model were 575 available in Lee et $\mathrm{al}^{57}$.

\section{Quantification of the phenotypic plasticity for the 23 agronomic traits}

577 Since all the 1404 maize lines were phenotyped for 23 agronomic traits across five sites, we 578 quantified and studied the genetics of maize complex trait plasticity in response to 579 longitudinal and latitudinal environmental variation. Here, the phenotypic plasticity was 580 classified into two categories (Figure S2B-E). The first category is overall plasticity 581 describing plasticity across all the studied environments, while the second category-specific 582 plasticity is more unique to certain pairs of sites, which only captures the plasticity across 583 two environments. The motivation underlying such classification is that some individuals are 584 more robust across most of the studied sites except only one or a few sites, while other 585 individuals are plastic among most of the sites.

586 One metric, pairwise difference in phenotypic value between two sites, was used to quantify 587 the specific plasticity (Figure S2A). Using DTT measured at JL and HN as an example, the 588 differences in measured DTT values for all individuals $\left(D I F F_{D T T}^{J L-H N}=\mathrm{DTT}_{\mathrm{JL}}-\mathrm{DTT}_{\mathrm{HN}}\right)$ would 589 describe the specific plasticity between $\mathrm{HN}$ and BJ (Figure S2B) ${ }^{31}$. In addition, four 590 additional approaches were used to quantify the overall plasticity (Figure S2C-E). First, the 591 principal component analysis (PCA) was used to quantify the overall plasticity. The influence 592 of the phenotype measures at individual sites on the principal components (PCs) can be 593 captured in the loadings ${ }^{58}$ (Figure S2C). As the second PC (PC2) captures more variation in 594 overall plasticity, we consider PC2 as a measure for overall phenotypic plasticity. Second, the 595 across environment variance (VAR) of the rank transformed phenotype proposed in Vanous 
596 et al., 2019 was used (Figure S2D), and the coefficient of variance (CV) ${ }^{7}$ was also used to

597 account for the mean difference. The fourth score for the overall plasticity (FWR) applies

598 Finlay-Wilkinson Regression ${ }^{32,59}$ to partition the phenotype into two components, one is 599 constant across environments and another responds dynamically to environmental changes.

600 Using the linear mixed model, the phenotype of each line is partitioned into these two 601 components and the plasticity component is used as a measurement of plasticity. In total, the 602 described approaches resulted in 14 measurements of phenotypic plasticity (abbreviated as 603 DIFF, PCA, VarR, CV, and FWR). Altogether, these three metrics yield 14 plasticity 604 measurements for each trait.

605 Genome-wide association analysis for the trait mean/median and plasticity measurements

606 To detect genetic polymorphisms underlying variation of agronomic trait mean and plasticity, 607 we fitted the following linear mixed model:

$608 \quad \mathrm{Y}=\mu+\mathrm{X} \beta+\mathrm{Zu}+\mathrm{e}(3)$

609 where $\mathrm{Y}, \mu, \mathrm{Z}, \mathrm{u}$, and e are the same as has been defined in the model (1). $\mathrm{X}$ is a matrix 610 containing the genotype of the tested SNP (coded as 0/2 for minor/major-allele homozygous 611 genotypes, respectively). $\beta$ is a vector including the estimated additive allele-substitution 612 effect for the tested SNP. First, a genome-wide analysis (GWA) across all genotyped SNPs 613 was conducted using GEMMA ${ }^{60}$. A subsequent conditional analysis was performed where all 614 the top associated SNPs (the SNPs with the highest P value from each association QTL from 615 the initial GWA scan) were included as covariates in the design matrix $\mathrm{X}$ to screen for 616 additional association signals. This conditional analysis was repeated until no more SNPs 617 were above the significance threshold. This conditional analysis was implemented in cojo 618 module from GCTA ${ }^{61}$. The linkage disequilibrium (LD) was high in this population, making 619 Bonferroni correction assuming all tested markers were statistically independent too 620 conservative. Therefore, we estimated the effective number of independent markers (Me) ${ }^{62}$ 621 and derived a less conservative genome-wide significance threshold following 0.05/Me (1.53 $622 \times 10^{-8}$ equivalent to $\left.-\log _{10}{ }^{\mathrm{p}}=7.81\right)$.

623 Colonization test separates linkage from pleiotropy at regions where multiple signals were 624 associated with multiple traits

625 At the same genomic region, multiple association signals, each associated with one or 626 multiple traits, were colocalized. Since the level of LD between the lead SNPs is very low, 627 we could not directly tell whether multiple independent signals, detected in multiple scans 
628 and physically close to each other, are from one association signal simultaneously associated

629 with multiple scans (pleiotropy), or multiple associations each associated with one scan but in 630 tight $\mathrm{LD}$ with each other. To distinguish this, we performed a multi-trait colocalization 631 analysis (Supplementary note). This method estimates a posterior probability of whether 632 multiple traits are sharing a common causal variant using summaries statistics from each 633 trait ${ }^{63,64}$. We first binned the genome into $1 \mathrm{Mb}$ bins. Scans with independent SNPs that fall 634 into consecutive bins were aggregated and tested for colocalization using the hyprcoloc $\mathrm{R}$ 635 package ${ }^{63,64}$. Given the complex population history (multi-parental) and a limited number of 636 recombinations, some of the statistically independent SNPs were very close to each other. To 637 make a comparison among the 4 types of measurements, we arbitrarily grouped SNPs less 638 than $1 \mathrm{Mb}$ to a single QTL.

\section{Gene-based test to prioritise candidate genes}

640 The LD was too extensive to directly pinpoint the genes underlying the associated loci. We, 641 therefore, applied a set-based analysis that aggregates summary statistics from all the variants $64250 \mathrm{~kb}$ up/downstream of the tested gene to obtain one $\mathrm{p}$ value to represent the significance of 643 a particular gene. The summary association statistics, including effect sizes, standard errors, 644 minor allele frequencies, and sample size, were first extracted from the GEMMA association 645 output, and subsequently inputted to fastBAT module in GCTA ${ }^{65}$. And 39,155 genes, 646 annotated in the B73 reference genome version 3 were used to bin the summary statistics to 647 perform the set analysis ${ }^{51}$.

\section{Testing for genotype by environment interaction of detected QTLs}

649 We tested the interaction between QTLs associated with each of the 23 traits in at least one of 650 the five sites, one QTL and one trait at a time. This was done by fitting the model below:

$651 \quad \bar{y}_{\mathrm{ij}}=\mathrm{u}+i d_{i}+\operatorname{site}_{\mathrm{j}}+Q T L_{i}+e_{i j}(4)$

$652 \quad \bar{y}_{\mathrm{ij}}=\mathrm{u}+i d_{i}+\operatorname{site}_{\mathrm{j}}+Q T L_{i} *$ site $_{j}+e_{i j}(5)$

653 This model was fitted for each of the 23 traits one at a time. $\bar{y}_{i j}$ is the trait mean/median of 654 individual $\mathrm{i}(\mathrm{i}=1 \ldots \mathrm{n}, \mathrm{n}=1404$, number of individuals) at site $\mathrm{j}$ ( $\mathrm{j}=1 \ldots \mathrm{q} ; \mathrm{q}=5$, corresponds to 655 the number of sites); $\mathrm{id}_{\mathrm{i}}$ is the line id (genotype) coded as a factor; site $_{\mathrm{j}}$ is a vector of 656 characters representing the site where the measurements were made. $Q T L_{i}$ is the genotype of $657 \mathrm{id}_{\mathrm{i}}$ at the testing QTL, and $Q T L_{i} *$ site $_{j}$ is the interaction terms (G-by-E) between a particular 658 QTL and the sites (environments). A likelihood ratio test comparing the model with (Model 4) 
659 and without (Model 5) the interaction between sites was performed to calculate $\mathrm{p}$ values. The 660 significance threshold was derived as 0.05 dividing the number of tests $\left(0.05 / 143=3.49 \times 10^{-}\right.$ $\left.661{ }^{04}\right)$.

\section{Experimental validation of maize flowering genes}

663 Knock-out lines of ZmTPS14.1 and ZmSPL6 were generated using a high-throughput 664 genome-editing system ${ }^{35}$. In brief, line-specific sgRNAs were filtered based on assembled 665 pseudo-genome of the receptor KN5585. The Double sgRNAs pool (DSP) approach was used 666 to construct vectors. The vectors were transformed into the receptor KN5585. The genotype 667 of gene-editing lines was identified by PCR amplification and Sanger sequencing using 668 target-specific primers (Table S13). The phenotype of knock-out lines and wild type were 669 investigated in Jilin (Gongzhuling, Jilin province, N $43^{\circ} 30^{\prime}$, E $124^{\circ} 49^{\prime}$ ) and Hainan (Sanya, 670 Hainan Province, N $18^{\circ} 34^{\prime}$, E $108^{\circ} 43^{\prime}$ ).

\section{Time-course transcriptome}

672 B73 seeds were planted at two conditions, long-day condition (14 hours light and 10 hours 673 dark) and short-day condition (8 hours light and 16 hours dark). Leaf tissues were harvested 674 at 3 time points in one day at stage V4 (Vegetative 4, four fully extended leaves). Eighteen 675 samples ( 2 conditions $\times 3$ time points $\times 3$ replicates) were RNA-sequenced by Hiseq 3000 . 676 Low-quality reads were filtered out by trimmomatic ${ }^{66} . \mathrm{STAR}^{67}$ was used to align the RNA677 seq reads to the reference genome. HTSeq ${ }^{68}$ was used to obtain gene-level counts from the 678 resulting BAM files. Genes with significant expression changes were detected by 679 ImpulseDE2 $2^{69}$.

680 Estimating the contribution from mean and plasticity QTLs to the variation of mean and 681 plasticity measurements

682 We quantified the contribution from mean and plasticity QTLs to the variation of trait mean 683 and plasticity by fitting the following models.

$684 \quad \mathrm{Y}=X_{1} \beta_{1}+X_{2} \beta_{2}+\mathrm{Zu}+\mathrm{e}(6)$

685 Here, $Y$ is a vector of length $n(n=1404)$, representing the trait mean or plasticity 686 measurement. The joint contributions from mean and plasticity QTLs were modelled in $X_{1} \beta_{1}$ 687 and $X_{2} \beta_{2}$ where $X_{1}$ and $X_{2}$ are the design matrixes $\beta_{1}$ and $\beta_{2}$ are the corresponding effect 688 sizes. $\mathrm{Z}, \mathrm{u}$ and $\mathrm{e}$ is the same as defined in model 3. Contributions from mean and plasticity 689 QTL were then calculated with $\operatorname{Var}_{\mathrm{m}}=\frac{\operatorname{Var}\left(X_{1} \beta_{1}\right)}{\operatorname{Var}(y)}$ and $\operatorname{Var}_{\mathrm{p}}=\frac{\operatorname{Var}\left(X_{2} \beta_{2}\right)}{\operatorname{Var}(y)}$. 
690 Forecasting the site-specific performance of the 23 traits

691 We fitted the following model to predict the performance of each site for the 23 traits one at a 692 time.

$693 \mathrm{Y}=X_{1} \beta_{1}+\mathrm{Zu}+\mathrm{e}(7)$

$694 \mathrm{Y}=X_{2} \beta_{2}+\mathrm{Zu}+\mathrm{e}(8)$

695 Here, $Y$ is a vector of length $n * p(n=1404$, the number of individuals; $p=5$, the number of 696 sites; $n * p=7020)$, representing the trait means measured at five sites. $u$ is a vector of length $697 \mathrm{n} * \mathrm{p}$, representing the breeding value of the $\mathrm{n}$ maize line, and e is the randomly distributed 698 residual with length $n^{*}$ p. The $Z$ matrix satisfies $Z Z^{\prime}=\mathrm{G} \otimes I$, where $\mathrm{G}$ is the identity by state 699 (IBS) matrix and I is a diagonal matrix of pxp. $X_{1}$ is a design matrix with one column of 1 700 representing column mean and additional 4 columns representing the environmental effects 701 from the remaining 4 sites, and $\beta_{1}$ is a vector of corresponding effect sizes. $X_{2}$ includes all 702 the columns from $X_{1}$ and additional columns with genotypes of the k QTL associated with 703 the mean and plasticity measures of the tested trait, and additional columns representing the 704 interaction between the k QTL and the five sites, capturing the effects from QTL by 705 environment factor interaction. The fitted values from model 7 were referred to as GBLUP 706 predictions while the fitted values from model 8 were referred to as GEAI predictions. These 707 models were fitted using $\operatorname{rrBLUP}^{70}$ package in $\mathrm{R}$ (https://www.R-project.org/). In the first 708 case, we evaluated the predictability on untested lines at any of the five sites by using all the 709 lines phenotyped across the five sites using 5-fold cross-validation. Each time, 80\% of the 710 lines were randomly sampled and used to predict the remaining $20 \%$ lines. In the second 711 case, we simulated a serial of more challenging breeding designs, in which only a core set of 712 lines $(10 \%-70 \%)$ were phenotyped across five sites and the interest was to predict the 713 performance of unphenotyped lines at each site. Each time, a core set of lines were randomly 714 sampled and the remaining lines were divided into 4 sets and were randomly assigned to one 715 of the remaining 4 sites, whose phenotypes were masked as NA and unassigned 716 environments. Accuracies were estimated as the regression $r^{2}$ between measured and 717 predicted phenotypes.

718

\section{Supplementary materials}

720 Figure S1-S10, Table S1-S13, and note were available in supplementary files 


\section{Author Contributions}

723 Conceptualization, J.Y., M.J., Y.Z., H.L.; methodology, Y.Z., M.J., H.L.; formal analysis, 724 Y.Z., M.J.; investigation, Y.Z., M.J., H.L.; data collection and curation, X.L., J.G., Y.Y., Z.L., 725 J.Z., X.W., F.Q., M.J., Y.Z., H.L.; writing original draft preparation, Y.Z., M.J.; writing 726 reviewing and editing, M.J., Y.Z., H.L., T.G., Y.X., J.Y.; visualization, Y.Z., M.J, Y.J;

727 supervision, J.Y.; funding acquisition, J.Y., Y.Z., H.L., X.L. All authors have read and agreed 728 to the published current version of the manuscript.

729

The authors have no conflicts of interest to declare.

\section{Funding}

733 This research was funded by the Natural Science Foundation of China (31961133002, 734 31901553, 31771879), the National Key Research and Development Program of China 735 (2020YFE0202300), the Swedish Research Council for Environment, Agricultural Sciences 736 and Spatial Planning to Y.Z. (2019-01600), and the Jilin Scientific and Technological 737 Development Program (20190201290JC).

\section{Acknowledgments}

740 We would like to thank the Swedish National Infrastructure for Computing (SNIC) for their 741 support in computation resources through High Performance Computing Centre North 742 (HPC2N, SNIC 2020/9-117) and the Uppsala Multidisciplinary Centre for Advanced 743 Computational Science (UPPMAX).

\section{Reference}

746 1. Sultan, S.E. Phenotypic plasticity for plant development, function and life history. $747 \quad$ Trends Plant Sci. 5, 537-42 (2000).

748 2. Nicotra, A.B. et al. Plant phenotypic plasticity in a changing climate. Trends Plant Sci. $749 \quad \mathbf{1 5}, 684-92(2010)$. 
750 3. Assmann, S.M. Natural variation in abiotic stress and climate change responses in Arabidopsis: implications for Twenty-first-century agriculture. Int J Plant Sci. 174, 3-26 (2013).

4. El-Soda, M., Malosetti, M., Zwaan, B.J., Koornneef, M. \& Aarts, M.G.M. Genotype $\times$ environment interaction QTL mapping in plants: lessons from Arabidopsis. Trends Plant Sci. 19, 390-8 (2014).

5. Sasaki, E., Zhang, P., Atwell, S., Meng, D. \& Nordborg, M. "Missing" G x E variation controls flowering time in Arabidopsis thaliana. PLoS Genet. 11, e1005597 (2015).

6. Schneider, J.R., Chadee, D.D., Mori, A., Romero-Severson, J. \& Severson, D.W. Heritability and adaptive phenotypic plasticity of adult body size in the mosquito Aedes aegypti with implications for dengue vector competence. Infect Genet Evol. 11, 11-6 (2011).

7. Vanous, A. et al. Stability analysis of kernel quality traits in exotic-derived doubled haploid maize lines. Plant Genome. 12, 1-14 (2019).

8. Mangin, B. et al. Genetic control of plasticity of oil yield for combined abiotic stresses using a joint approach of crop modelling and genome-wide association. Plant Cell Environ. 40, 2276-91 (2017).

9. Pigliucci, M. Evolution of phenotypic plasticity: where are we going now? Trends Ecol Evol. 20, 481-6 (2005).

10. Kang, M.S. Using genotype-by-environment interaction for crop cultivar development. Adv Agron. 62, 199-252 (1997).

11. Kusmec, A., de Leon, N. \& Schnable, P.S. Harnessing phenotypic plasticity to improve maize yields. Front Plant Sci. 9, 1377 (2018).

12. Gage, J.L. et al. The effect of artificial selection on phenotypic plasticity in maize. Nat Commun. 8, 1348 (2017).

13. Lynch, M. \& Walsh, B. Genetics and analysis of quantitative traits. Sinauer Assoc. 1 Ed, 980p (1998).

14. Jannink, J-L., Lorenz, A.J. \& Iwata, H. Genomic selection in plant breeding: from theory to practice. Brief Funct Genomics. 9, 166-77 (2010).

15. Blanquart, F., Kaltz, O., Nuismer, S.L. \& Gandon, S. A practical guide to measuring local adaptation. Ecol Lett. 16, 1195-205 (2013).

16. Hereford, J. A quantitative survey of local adaptation and fitness trade-offs. Am Nat. 173, 579-88 (2009). 
17. Anderson, J.T., Lee, C-R., Rushworth, C.A., Colautti, R.I. \& Mitchell-Olds, T. Genetic trade-offs and conditional neutrality contribute to local adaptation. Mol Ecol. 22, 699708 (2013).

18. Lazzaro, B.P., Flores, H.A., Lorigan, J.G. \& Yourth, C.P. Genotype-by-environment interactions and adaptation to local temperature affect immunity and fecundity in Drosophila melanogaster. PLoS Pathog. 4, e1000025 (2008).

19. Malosetti, M., Ribaut, J-M. \& van Eeuwijk, F.A. The statistical analysis of multienvironment data: modeling genotype-by-environment interaction and its genetic basis. Front Physiol. 4, 44 (2013).

20. Finlay, K. \& Wilkinson, G. The analysis of adaptation in a plant-breeding programme. Aust J Agric Res. 14, 742-754 (1963).

21. Gollob, H.F. A statistical model which combines features of factor analytic and analysis of variance techniques. Psychometrika. 33, 73-115 (1968).

22. Jiang, C. \& Zeng, Z.B. Multiple trait analysis of genetic mapping for quantitative trait loci. Genetics. 140, 1111-27 (1995).

23. Rauw, W.M. \& Gomez-Raya, L. Genotype by environment interaction and breeding for robustness in livestock. Front Genet. 6, 310 (2015).

24. Kusmec, A., Srinivasan, S., Nettleton, D. \& Schnable, P.S. Distinct genetic architectures for phenotype means and plasticities in Zea mays. Nat plants. 3, 715-23 (2017).

25. $\mathrm{Li}, \mathrm{C}$. et al. Genetic architecture of phenotypic means and plasticities of kernel size and weight in maize. Theor Appl Genet. 132, 3309-20 (2019).

26. Schneider, H.M. et al. Genetic control of root architectural plasticity in maize. J Exp Bot. 71, 3185-97 (2020).

27. Liu, N., Du, Y., Warburton, M.L., Xiao, Y.\& Yan, J. Phenotypic plasticity contributes to maize adaptation and heterosis. Mol Biol Evol. 38, 1262-75 (2021).

28. Li, X., Guo, T., Mu, Q., Li, X. \& Yu, J. Genomic and environmental determinants and their interplay underlying phenotypic plasticity. Proc Natl Acad Sci U S A. 115, 6679-84 (2018).

29. Liu, H-J. et al. CUBIC: an atlas of genetic architecture promises directed maize improvement. Genome Biol. 21, 20 (2020).

30. Zan, Y. \& Carlborg, Ö. Dissecting the genetic regulation of yeast growth plasticity in tesponse to environmental changes. Genes (Basel). 11, 1279 (2020). 
815 31. Ungerer, M.C., Halldorsdottir, S.S., Purugganan, M.D. \& Mackay, T.F.C. Genotype816 environment interactions at quantitative trait loci affecting inflorescence development in 817 Arabidopsis thaliana. Genetics. 165, 353-65 (2003).

818 32. Lian, L. \& de Los Campos, G. FW: An R package for Finlay-Wilkinson regression that 819 incorporates genomic/pedigree information and covariance structures between 820 environments. G3 (Bethesda). 6, 589-97 (2015).

821 33. Balasubramanian, S. et al. The PHYTOCHROME C photoreceptor gene mediates natural 822 variation in flowering and growth responses of Arabidopsis thaliana. Nat Genet. 38, $711-$ $823 \quad 5(2006)$.

824 34. Kim, S.Y., Yu, X. \& Michaels, S.D. Regulation of CONSTANS and FLOWERING 825 LOCUS T expression in response to changing light quality. Plant Physiol. 148, 269-79 826 (2008).

827 35. Liu, H-J. et al. High-throughput CRISPR/Cas9 mutagenesis streamlines trait gene 828 identification in maize. Plant Cell. 32, 1397-413 (2020).

829 36. Sommer, R.J. Phenotypic plasticity: from theory and genetics to current and future 830 challenges. Genetics. 215, 1-13 (2020).

831 37. Tsai, A. Y-L. \& Gazzarrini, S. Trehalose-6-phosphate and SnRK1 kinases in plant 832 development and signaling: the emerging picture. Front Plant Sci. 5, 119 (2014).

833 38. Andrés, F. \& Coupland, G. The genetic basis of flowering responses to seasonal cues. $834 \quad$ Nat Rev Genet. 13, 627-39 (2012).

835 39. Fitter, A.H. \& Fitter, R.S.R. Rapid changes in flowering time in British plants. Science. 836 296, 1689-91 (2002).

837 40. Blázquez, M.A., Ahn, J.H. \& Weigel, D. A thermosensory pathway controlling flowering 838 time in Arabidopsis thaliana. Nat Genet. 33, 168-71 (2003).

839 41. Li, X. et al. An integrated framework reinstating the environmental dimension for 840 GWAS and genomic selection in crops. Mol Plant. 14, 874-87 (2021).

841 42. Nagano, A.J. et al. Annual transcriptome dynamics in natural environments reveals plant 842 seasonal adaptation. Nat Plants. 5, 74-83 (2019).

843 43. Yoshida, S. Fundamentals of rice crop science. IRRI, Los Baños, Philippines, p. 269 844 (1981).

845 44. Wallace, D.H., Zobel, R.W. \& Yourstone, K.S. A whole-system reconsideration of 846 paradigms about photoperiod and temperature control of crop yield. Theor Appl Genet.

$847 \quad \mathbf{8 6}, 17-26(1993)$. 
848

849

850

851

852

853

854

855

856

857

858

859

860

861

862

863

864

865

866

867

868

869

870

871

872

873

874

875

876

877

878

879

880

45. McFarland, B.A. et al. Maize genomes to fields (G2F): 2014-2017 field seasons: genotype, phenotype, climatic, soil, and inbred ear image datasets. BMC Res Notes. 13, 71 (2020).

46. D’Andrea, K.E., Otegui, M.E., Cirilo, A.G. \& Eyhérabide, G.H. Parent-progeny relationships between maize inbreds and hybrids: analysis of grain yield and its determinants for contrasting soil nitrogen conditions. Crop Sci. 53, 2147-61 (2013).

47. Prado, S.A., Sadras, V.O. \& Borrás, L. Independent genetic control of maize (Zea mays L.) kernel weight determination and its phenotypic plasticity. J Exp Bot. 65, 4479-87 (2014).

48. Des Marais, D.L., Hernandez, K.M. \& Juenger, T.E. Genotype-by-environment interaction and plasticity: exploring genomic responses of plants to the abiotic environment. Annu Rev Ecol Evol Syst. 44, 5-29 (2013).

49. Zan, Y. \& Carlborg, Ö. Dynamic genetic architecture of yeast response to environmental perturbation shed light on origin of cryptic genetic variation. PLoS Genet. 16, e1008801 (2020).

50. Wang, B. et al. Genome-wide selection and genetic improvement during modern maize breeding. Nat Genet. 52, 565-71 (2020).

51. Law, M. et al. Automated update, revision, and quality control of the maize genome annotations using MAKER-P improves the B73 RefGen_v3 gene models and identifies new genes. Plant Physiol. 167, 25-39 (2015).

52. Meng, X., Muszynski, M.G. \& Danilevskaya, O.N. The FT-like ZCN8 gene functions as a floral activator and is involved in photoperiod sensitivity in maize. Plant Cell. 23, 94260 (2011).

53. Murakami, M., Yamashino, T. \& Mizuno, T. Characterization of circadian-associated APRR3 pseudo-response regulator belonging to the APRR1/TOC1 quintet in Arabidopsis thaliana. Plant Cell Physiol. 45, 645-50 (2004).

54. Kumar, S.V. et al. Transcription factor PIF4 controls the thermosensory activation of flowering. Nature. 484, 242-5 (2012).

55. Matsoukas, I.G., Massiah, A.J. \& Thomas, B. Starch metabolism and antiflorigenic signals modulate the juvenile-to-adult phase transition in Arabidopsis. Plant Cell Environ. 36, 1802-11 (2013).

56. Yang, J., Lee, S.H., Goddard, M.E. \& Visscher, P.M. GCTA: a tool for genome-wide complex trait analysis. Am J Hum Genet. 88, 76-82 (2011). 
881

57. Lee, S.H., Yang, J., Goddard, M.E., Visscher, P.M. \& Wray, N.R. Estimation of pleiotropy between complex diseases using single-nucleotide polymorphism-derived genomic relationships and restricted maximum likelihood. Bioinformatics. 28, 2540-2 (2012).

58. Yano, K. et al. GWAS with principal component analysis identifies a gene comprehensively controlling rice architecture. Proc Natl Acad Sci U S A. 116, 21262-7 (2019).

59. Weber S.L. \& Scheiner S.M. The genetics of phenotypic plasticity. IV. Chromosomal localization. J Evol Biol. 5, 109-20 (1992).

60. Zhou, X. \& Stephens, M. Genome-wide efficient mixed-model analysis for association studies. Nat Genet. 44, 821-4 (2012).

61. Yang, J. et al. Conditional and joint multiple-SNP analysis of GWAS summary statistics identifies additional variants influencing complex traits. Nat Genet. 44, 369-75 (2012).

62. Li, M-X., Yeung, J.M.Y., Cherny, S.S. \& Sham, P.C. Evaluating the effective numbers of independent tests and significant p-value thresholds in commercial genotyping arrays and public imputation reference datasets. Hum Genet. 131, 747-56 (2012).

63. Berisa, T. \& Pickrell, J.K. Approximately independent linkage disequilibrium blocks in human populations. Bioinformatics. 32, 283-5 (2016).

64. Foley, C.N. et al. A fast and efficient colocalization algorithm for identifying shared genetic risk factors across multiple traits. Nat Commun. 12, 764 (2021).

65. Bakshi, A. et al. Fast set-based association analysis using summary data from GWAS identifies novel gene loci for human complex traits. Sci Rep. 6, 32894 (2016).

66. Bolger, A.M., Lohse, M. \& Usadel, B. Trimmomatic: a flexible trimmer for Illumina sequence data. Bioinformatics. 30, 2114-20 (2014).

67. Dobin, A. et al. STAR: ultrafast universal RNA-seq aligner. Bioinformatics. 29, 15-21 (2013).

68. Anders, S., Pyl, P.T. \& Huber, W. HTSeq--a Python framework to work with highthroughput sequencing data. Bioinformatics. 31, 166-9 (2015).

69. Fischer, D.S., Theis, F.J. \& Yosef, N. Impulse model-based differential expression analysis of time course sequencing data. Nucleic Acids Res. 46, e119 (2018).

70. Endelman, J.B. Ridge regression and other kernels for genomic selection with R package rrBLUP. Plant Genome. 4, 250-5 (2011). 